\title{
Anemia y depleción de las reservas de hierro en adolescentes gestantes de una zona urbana y rural del estado Zulia, Venezuela
}

\author{
Anemia and iron depleted reserves in \\ pregnant adolescents from urban and rural area, \\ Zulia state, Venezuela
}

\begin{abstract}
The objective of the present study was to analyze the prevalence of anemia and of iron deposits depletion (FeD) in pregnant adolescents from an urban $(U)$ and rural $(R)$ area. Methods: We studied 214 pregnant adolescents $(U=100 ; R=114)$, from low socio-economic status. Clinical obstetric, nutritional situation and biochemical assessment was performed. Anemia in pregnant adolescents was determined during the first and third trimesters when $\mathrm{Hb} \leq 110 \mathrm{~g} / \mathrm{L}$, second trimester $\mathrm{Hb} \leq 105 \mathrm{~g} / \mathrm{L}$. FeD: ferritin $<15 \mu \mathrm{g} / \mathrm{L}$; insufficient reserves of iron $I R I=15-20 \mu \mathrm{g} / \mathrm{L}$ and normal reserves of iron $(R I N)>20 \mu \mathrm{g} / \mathrm{L}$, as recommended by $\mathrm{WHO}$ and the International Anemia Consultative Group. The statistical significance was considered when $p<0.05$. Results: The prevalence of anemia in pregnant adolescents with depleted reserves of iron (FeD+IRI) was $45.79 \%(R=22.90 \%$ vs $U=22.89 \%)$; reserves of iron without anemia was $30.37 \%$. ( $R=17.29 \%$ vs. $U=13.08 \%)$; and anemia with RIN was $9.34 \%(R=6.07 \%$ vs. $U=3.27 \%)$; there was a statistically significant association to urban $(2=8.1282 p=0.0172)$ and rural area $(2=11.6270 p=0.0030)$. Conclusion: Iron deficiency anemia is a common complication in pregnant adolescents from rural and urban areas. This situation is associated with nutritional bad habits in adolescents. Strategies for recovery nutrition and education in primary health care and maternal health programs are needed. Key words: Anemia; Iron deficiency; adolescents; pregnancy.
\end{abstract}

\section{INTRODUCCIÓN}

La depleción de las reservas corporales de hierro (DRFe) y la anemia por deficiencia de hierro son problemas nutricionales de salud pública. La OMS estima que en el mundo existen aproximadamente 2.000 millones de personas anémicas, y cerca de $50 \%$ de los casos pueden atribuirse a la carencia de hierro (1-3), observándose mayor prevalencia en los países de América Latina y el Caribe en proceso de transición demográfica, epidemiológica y nutricional, siendo los más afectados lactantes, adolescentes y mujeres en edad fértil de estratos socioeconómicos bajos que residen en zonas periurbanas y rurales (3-5).

En Venezuela, la prevalencia de anemia es variable, oscila entre $14,9 \%$ y $78.0 \%(6,7)$ y de DRFe en niños y adolescentes, varía entre $9 \%$ y $34,7 \%(6,7)$. Entre las causas de anemia reportadas destacan la malnutrición pluricarencial y de micronutrientes, seguida por procesos infecciosos o infla-
Pablo Antonio Ortega F. Jorymar Yoselyn Leal M.

Carlos Javier Chávez Lidia Mejías C.

Noraima Chirinos Q.

Carolina del Pilar Escalona V.

Laboratorio de Investigación en Malnutrición Infantil. Instituto de Investigaciones Biológicas. Facultad de Medicina. Universidad del Zulia. Maracaibo, Venezuela.

Dirigir la correspondencia a: Profesor

Pablo Ortega $F$

Laboratorio de Investigación en Malnutrición Infantil. Instituto de Investigaciones Biológicas. Facultad de Medicina. Universidad del Zulia

Apartado 526. Maracaibo, 4001 Venezuela.

Teléfono: $+582614172033,+582616357496$ E-mail: portega22@cantv.ne portega@luz.edu.v iyleal@hotmail.com

Este trabajo fue recibido el 3 de Julio de 2012 y aceptado para ser publicado el 5 de Agosto de 2012.

matorios prolongados, pérdida crónica de sangre y aumento de los requerimientos ocasionados por el crecimiento, embarazo y lactancia (8-11).

Se ha reportado, que la anemia por deficiencia de hierro es responsable entre $75 \%$ a $90 \%$ de todas las anemias diagnosticadas durante el embarazo (12). La mayoría de los estudios demuestran una frecuencia superior de anemia en adolescentes embarazadas que en otros grupos de edades, debido al incremento en la demanda de hierro como consecuencia del crecimiento acelerado de la madre y los tejidos fetales, lo cual frecuentemente coexiste con desnutrición e insuficiente ganancia de peso durante la gestación, factores que incrementan la morbimortalidad materna y perinatal (12-15).

En América Latina, se calcula que $3 \%$ de las muertes maternas son atribuibles directamente a la anemia y se asocia con aumento del riesgo de infección, fatiga y mayores pérdidas sanguíneas durante el parto y puerperio $(16,17)$. 
La anemia es un indicador de malnutrición y enfermedad; sin embargo, es muy común que aproximadamente $25 \%$ de las mujeres jóvenes, aparentemente sanas, en su primera visita prenatal presenten DRFe sin síntomas de anemia, pudiendo esta situación comprometer la salud materna y fetal (18). Justamente, la anemia es el indicador comúnmente utilizado para estimar la DRFe; no obstante, considerar el estado del hierro con base en la presencia de anemia puede ocasionar errores diagnósticos, debido a que durante las primeras etapas de DRFe las concentraciones de hemoglobina continúan por encima del valor límite determinado para anemia (18).

Considerando la anemia y la DRFe como indicativos clínicos de enfermedad en adolescentes gestantes, conocer su prevalencia en zonas urbanas y rurales puede contribuir en el desarrollo de programas de educación y prevención nutricional de la enfermedad. En este contexto, el objeto de este estudio fue analizar la prevalencia de anemia y DRFe en adolescentes gestantes, de baja condición socio-económica provenientes de zonas urbanas y rurales del estado Zulia, Venezuela.

\section{SUJETOS Y MÉTODO}

Se realizó un estudio transversal y descriptivo. La muestra estuvo constituida por 214 adolescentes gestantes, quienes acudían a consulta pre-natal del Servicio de Gineco-obstetricia del Hospital Central de Maracaibo "Dr. Urquinaona", dependiente del Ministerio de Salud y Desarrollo Social del Gobierno venezolano. Las adolescentes gestantes del municipio Maracaibo fueron consideradas como procedentes de la zona urbana (centros poblados con 2.500 habitantes y más). Las adolescentes gestantes de los municipios Mara y Páez fueron consideradas procedentes de la zona rural considerándose como centros poblados con menos de 1.000 habitantes y población diseminada (19).

Para la determinación del tamaño de la muestra se utilizó la expresión:

$$
n=\frac{N Z^{2} \sigma^{2}}{\left(E^{2}-1\right) N+Z^{2} \sigma^{2}}
$$

Donde:

$N$ : es el tamaño estimado de la población $=2850$ adolescentes gestantes.

Z: valor extraído de la tabla de la distribución normal para el $95 \%$ de confianza $=1,96$.

62: varianza estimada de la población con base en el valor mínimo y el valor máximo de hemoglobina 134 y 98, respectivamente.

Fue seleccionado un error máximo permisible de $10 \%$. Obteniendo un tamaño de muestra de $n=133$ adolescentes gestantes, las que fueron seleccionadas por el método aleatorio simple.

El estudio cumplió con lo dispuesto en las normas internacionales de ética para la investigación en humanos, por lo que fue aprobado por las Comisiones Científicas del Consejo de Desarrollo Científico y Humanístico de la Universidad del Zulia y el Fondo Nacional para el Avance de la Ciencia y Tecnología de la República Bolivariana de Venezuela (N²000-001904). Los padres o representantes legales de las adolescentes, una vez informados del objeto, beneficio y riesgos de la investigación, dieron su consentimiento verbal y escrito para su inclusión en el proyecto de investigación.

La evaluación socio-económica fue realizada utilizando el método de Graffar, modificado y adaptado para Venezuela por Méndez Castellano y Méndez (20). La evaluación clínica- obstétrica fue ejecutada por médicos supervisados por una especialista en ginecología y obstetricia, para determinar la situación obstétrica y detectar signos y síntomas sugestivos de déficit nutricional y anemia. Las adolescentes con antecedentes y/o síntomas de procesos infecciosos recientes fueron excluidas.

La evaluación nutricional-antropométrica fue realizada considerando las variables edad (E), peso $(P)$, talla ( $T)$, Índice de Masa corporal $(I M C=$ Peso/Talla2), Circunferencia Media del Brazo Izquierdo (CMBI) y el pliegue cutáneo tricipital (Ptr). Estos dos últimos permitieron evaluar las reservas energéticas y proteicas de las adolescentes. El porcentaje de déficit en las reservas proteíca y energética fue considerado cuando los valores se ubicaron por debajo del percentil 10 (21). Para la evaluación del crecimiento fetal se utilizaron las variables altura uterina (AU), ganancia de peso (GP) y edad gestacional (EG), siguiendo las recomendaciones de la OMS y OPS (22).

La evaluación dietética fue realizada mediante una encuesta de recordatorio de 24 horas y de frecuencia de consumo de alimentos de una semana $(23,24)$, utilizando la técnica de la entrevista al propio sujeto y llevada a cabo por el equipo de nutricionista-dietista Un vez obtenida la entrevista se codificaron los datos del consumo de alimentos según la Tabla de composición de Alimentos del Instituto Nacional de Nutrición, revisión 1.999 (25).

Se tomó una muestra de sangre $(5 \mathrm{ml})$, por punción venosa periférica en ayunas. La sangre fue colectada en dos tubos, uno con anticoagulante para la determinación de parámetros hematológicos, y otro sin anticoagulante sometido a centrifugación a $3000 \mathrm{rpm}$ durante 10 minutos para la determinación de hierro y ferritina sérica. La hemoglobina $(\mathrm{Hb})$, hematocrito (Hto) e índices eritrocitarios, fueron medidos en un contador hematológico electrónico automatizado marca Sysmex K-800. La evaluación del perfil hematológico se realizó según las recomendaciones de la OMS y el Grupo Consultivo Internacional de Anemia, considerándose como anemia en el I y III trimestre valores menores a $110 \mathrm{~g} / \mathrm{L}$, y durante el II trimestre valores menores a $105 \mathrm{~g} / \mathrm{L}$ (26). La determinación de la concentración de la ferritina sérica se realizó mediante la técnica de inmunoanálisis de micropartículas ligada a enzima (MIA) en un equipo IMX (Abbot). Se consideró depleción de las reservas corporales de hierro (DRFe) a valores de ferritina $<15 \mathrm{ug} / \mathrm{L}$; reservas insuficientes de hierro (RIFe) a una ferritina entre 15 y 20 ug/L y reservas de hierro normal (RFe normal) a ferritina >20 ug/L. La determinación del hierro sérico se realizó en un equipo de química sanguínea automatizado marca Stat Fax (1904 Plus), mediante el método colorimétrico sin desproteinización; tomándose como valores bajos a los $<60$ ug/dL $(27,28)$.

El procesamiento de los datos se realizó con el programa de Sistema de Análisis Estadístico (SAS) Versión 8.1 (SAS Institute, Cary, NC, USA 2000. Las variables ferritina y hierro sérico mostraron una distribución asimétrica, por lo que fueron normalizadas mediante transformación logarítmica. Para estas variables se calculó el promedio geométrico, al cual se le realizó el análisis estadístico respectivo. Se utilizó la prueba de X2. Para comparar las medias de los grupos se empleó el Análisis de la Varianza (ANOVA). Se consideró el 95\% como índice de confiabilidad estadística con una $\mathrm{p}<0,05$.

\section{RESULTADOS}

La tabla 1 muestra las características nutricionales, antropométricas, dietéticas y hematológicas de la población gestante estudiada, según su procedencia geográfica. Nótese 
que las adolescentes gestantes de la zona rural mostraron valores promedio de edad, peso previo al embarazo, peso actual, talla, frecuencia de consumo de hierro, así como de hierro sérico significativamente más bajos que las gestantes de la zona urbana.

En la tabla 2 se presenta la distribución de las adolescentes gestantes según la prevalencia de anemia con afectación de las reservas corporales de hierro RIFe+DRFe (Total=45,79\%; rurales $=22,90 \%$ vs urbanas $=22,89 \%$ ), afectación de las reservas corporales de hierro RIFe+DRFe sin anemia (Total=30,37\%; rurales $=17,29 \%$ vs. urbanas $=13,08 \%$ ) y anemia con reservas corporales de hierro normal (Total $=9,34 \%$; rurales $=6,07 \%$ vs. urbanas=3,27\%); mostrando asociación estadísticamente significativa según la distribución por zona geográfica de procedencia.

La tabla 3 muestra la distribución de las adolescentes gestantes según la edad gestacional en trimestre y zona geográfica de procedencia. Nótese que la prevalencia de anemia con afectación de las reservas de hierro fue mayor en las adolescentes urbanas al compararlas con las rurales del III trimestre (urbanas $=64,70 \%$ vs rurales $=61,37 \%$ ); seguido por las gestantes ubicadas en el II (urbanas $=47,92 \%$ vs rurales $=35,09 \%$ ) y I Trimestre de gestación (urbanas $=22,22 \%$ vs. rurales $=15,38 \%)$. Contrario a lo observado en relación con la prevalencia de afectación de las reservas de hierro (RIFe+DRFe) sin anemia, la cual fue superior en las gestantes rurales al compararlas con las gestantes de la zona urbana en el II y I trimestres (rurales $=38,60 \%$ vs. urbana $=31,25 \%$ y rurales $=38,45 \%$ vs. urbanas $=16,67 \%$, respectivamente); sin embargo, durante el III trimestre la prevalencia fue mayor en las adolescentes gestantes urbanas $(29,42 \%)$ que en las procedentes de la zona rural $(22,72 \%)$.

\section{DISCUSIÓN}

En el presente estudio, se detectó que las adolescentes gestantes procedentes de la zona rural presentan valores promedio significativamente más bajos con respecto a edad, peso previo al embarazo, peso actual y talla, cuando se comparan

\section{TABLA 1}

Características nutricionales, antropométricas y dietéticas de un grupo de adolescentes gestantes urbanas y rurales del estado Zulia-Venezuela.

Características

Nutricionales-antropométricas

Edad (años)

Peso pre-gestacional $(\mathrm{Kg})$

Peso gestacional $(\mathrm{Kg})$

Talla $(\mathrm{cm})$

CMB (cm)

IMC pre-gestacional $\left(\mathrm{Kg} / \mathrm{m}^{2}\right)$

IMC gestacional $\left(\mathrm{Kg} / \mathrm{m}^{2}\right)$

Edad gestacional (sem)

Altura Uterina (cm)

Ganancia de Peso (Kg)

Área muscular (mm)

Área grasa (mm)

Dietéticas

Recordatorio de 24 horas

Energía (Kcal)

Proteína (g)

Hierro (mg)

Frecuencia de consumo en 7 días

Energía (Kcal)

Proteína (g)

Hierro (mg)

Valores Hematológicos

Hemoglobina (g/L)

Hematócrito (\%)

VCM (fL)

$\mathrm{HCM}$ (pg)

CCMH $(\mathrm{g} / \mathrm{dL})$

Hierro

Hierro sérico (ug/dL)

Ferritina sérica (ug/L )
Zona geográfica de procedencia Urbana $(n=100)$

$$
\begin{gathered}
17,52 \pm 1,28 \\
52,06 \pm 8,59 \\
58,05 \pm 9,55 \\
156,88 \pm 5,88 \\
24,11 \pm 2,96 \\
21,14 \pm 3,25 \\
23,57 \pm 3,56 \\
24,15 \pm 8,43 \\
21,38 \pm 8,64 \\
5,99 \pm 6,66 \\
2929,12 \pm 596,20 \\
1162,05 \pm 489,83
\end{gathered}
$$

$76,60 \pm 28,41$

$73,62 \pm 28,04$

$41,72 \pm 18,04$

$66,53 \pm 21,90$

$63,52 \pm 21,55$

$52,32 \pm 36,27$

$106,28 \pm 11,34$

$31,96 \pm 2,93$

$81,69 \pm 6,13$

$27,17 \pm 2,39$

$33,23 \pm 1,13$

$77,94 \pm 43,89$

$14,00 \pm 11,59$
Rural $(n=114)$

$$
\begin{gathered}
16,73 \pm 1,47^{* *} \\
49,15 \pm 7,38^{*} \\
54,86 \pm 7,51^{*} \\
153,04 \pm 5,92^{* *} \\
23,69 \pm 2,03 \\
20,96 \pm 2,70 \\
23,41 \pm 2,83 \\
25,04 \pm 8,18 \\
22,51 \pm 7,60 \\
5,71 \pm 5,20 \\
2825,42 \pm 431,41 \\
1095,56 \pm 311,75
\end{gathered}
$$

$80,20 \pm 30,91$

$70,34 \pm 29,96$

$41,79 \pm 18,23$

$69,44 \pm 22,29$

$62,51 \pm 24,74$

$38,53 \pm 13,87^{* *}$

$105,53 \pm 11,07$

$31,63 \pm 3,07$

$81,52 \pm 4,61$

$27,23 \pm 1,82$

$33,38 \pm 0,92$

$62,31 \pm 37,85^{*}$

$13,16 \pm 11,77$

Los valores están expresados en $\mathrm{X} \pm \mathrm{DE}, I M C=$ Índice de Masa corporal,

VCM = Volumen Corpuscular Medio, $\mathrm{HCM}=$ Hemoglobina Corpuscular Media

$\mathrm{CCMH}=$ Concentración de hemoglobina Corpuscular Media

${ }^{*}=p<0,01^{* *}=p<0,001$ 
con gestantes procedentes de zonas urbanas. En relación a nuestros hallazgos, el embarazo en adolescentes en condiciones socio-demográficas precarias, dietas restrictivas, perturbaciones de la conducta alimentaria y bajo nivel educativo son los principales factores asociados a una escasa ganancia de peso gestacional en gestantes que habitan en zonas rurales. Al respecto, las adolescentes gestantes constituyen un grupo de riesgo y requieren generalmente una intervención nutricional en etapas precoces del embarazo, mientras menor sea el período post-menarquia de la gestación aumenta el riesgo de desnutrición debido a que sus necesidades de nutrientes y energía son mayores como consecuencia de no haber culminado su crecimiento $(4,31-34)$.

Estos resultados señalan que las adolescentes gestantes de la zona rural presentan valores promedio de frecuencia de consumo de hierro, así como de hierro sérico significativamente más bajos que las gestantes procedentes de la zona urbana. Resultados similares fueron reportados por Vila y Quintana, quienes observaron que $86,8 \%$ de las adolescentes evaluadas presentaron riesgo elevado de ingesta inadecuada de hierro (35). Estos hallazgos indican que existe un alto riesgo de deficiencia de hierro y anemia, como consecuencia del consumo insuficiente y la escasa biodisponibilidad del mismo en las dietas.

Los requerimientos aumentados durante el embarazo no pueden ser compensados únicamente por la dieta, por lo que organizaciones científicas internacionales y nacionales recomiendan la suplementación sistemática preventiva al inicio de la gestación con dosis de hierro entre 30 y 60 mg/día (36). Sin embargo, un gran porcentaje de mujeres de bajos recursos económicos y habitantes de zonas rurales en Venezuela no inician la administración preventiva de suplementos de hierro en el momento y pauta prescrita por el gineco-obstetra. Cabe enfatizar, que los requerimientos de hierro varían a lo largo de la gestación pero son especialmente altos en el segundo y tercer trimestres, debido a las mayores demandas maternas para incrementar el volumen eritrocitario, la placenta en desarrollo y el aumento de las necesidades fetales por el crecimiento, metabolismo y depósitos celulares $(33,37)$.

En la secuencia de etapas que van desde un estado nutricional normal del hierro hasta la anemia, algunos marcadores bioquímicos son más sensibles que otros para tomar medidas preventivas que permitan evitar la anemia por deficiencia de este mineral. El estadio inicial de la depleción se refleja en la

TABLA 2

Prevalencia de la DRFe en una poblacion de adolescentes gestantes según la zona geográfica de procedencia.

\begin{tabular}{|c|c|c|c|c|c|c|c|c|}
\hline & \multicolumn{2}{|c|}{ URBANA } & \multicolumn{2}{|c|}{ RURAL } & \multicolumn{2}{|c|}{ TOTAL } & \multirow[t]{2}{*}{$\times 2$} & \multirow[t]{2}{*}{$P$} \\
\hline & $\mathrm{n}$ & $\%$ & $\mathrm{n}$ & $\%$ & $\mathrm{n}$ & $\%$ & & \\
\hline \multicolumn{9}{|c|}{ Hb Normal } \\
\hline RFeN & 16 & 7,49 & 15 & 7,01 & 31 & 14,50 & & \\
\hline $\mathrm{RIFe}$ & 7 & 3,27 & 11 & 5,14 & 18 & 8,41 & 8,1282 & 0,0172 \\
\hline DRFe & 21 & 9,81 & 26 & 12,15 & 47 & 21,96 & & \\
\hline \multicolumn{9}{|l|}{ Anemia } \\
\hline RFeN & 7 & 3,27 & 13 & 6,07 & 20 & 9,34 & 11,6270 & 0,0030 \\
\hline $\mathrm{RIFe}$ & 10 & 4,67 & 2 & 0,94 & 12 & 5,61 & & \\
\hline DRFe & 39 & 18,22 & 47 & 21,96 & 86 & 40,18 & & \\
\hline TOTAL & 100 & 46,73 & 114 & 53,27 & 214 & 100,0 & & \\
\hline
\end{tabular}

$\mathrm{Hb}=$ Hemoglobina, RFeN= Reservas de hierro normales, RIFe= Riesgo de depleción de las reservas de hierro,

$\mathrm{DRFe}=$ Depleción de las reservas corporales de hierro.

TABLA 3

Prevalencia de anemia y DRFe en adolescentes gestantes, según zona geografica de procedencia y edad gestacional.

\begin{tabular}{|c|c|c|c|c|c|c|}
\hline & \multicolumn{2}{|c|}{$<14$ semanas } & \multicolumn{2}{|c|}{$14->28$ semanas } & \multicolumn{2}{|c|}{$>28$ semanas } \\
\hline & $\begin{array}{l}\text { Urbana } \\
(n=18)\end{array}$ & $\begin{array}{c}\text { Rural } \\
(n=13)\end{array}$ & $\begin{array}{l}\text { Urbana } \\
(n=48)\end{array}$ & $\begin{array}{c}\text { Rural } \\
(n=57)\end{array}$ & $\begin{array}{l}\text { Urbana } \\
(n=34)\end{array}$ & $\begin{array}{c}\text { Rural } \\
(n=44)\end{array}$ \\
\hline \multicolumn{7}{|c|}{ Hb Normal } \\
\hline RFeN & $8(44,44)$ & $4(30,77)$ & $6(12,50)$ & $8(14,03)$ & $2(5,88)$ & $3(6,82)$ \\
\hline $\mathrm{RIFe}$ & $1(5,56)$ & $3(23,07)$ & $3(6,25)$ & $7(12,28)$ & $3(8,82)$ & $1(2,27)$ \\
\hline DRFe & $2(11,11)$ & $2(15,38)$ & $12(25,00)$ & $15(26,32)$ & $7(20,60)$ & $9(20,45)$ \\
\hline \multicolumn{7}{|l|}{ Anemia } \\
\hline RFeN & $3(16,67)$ & $2(15,38)$ & $4(8,33)$ & $7(12,28)$ & 0 & $4(9,09)$ \\
\hline RIFe & $2(11,11)$ & $1(7,69)$ & $5(10,42)$ & 0 & $3(8,82)$ & $1(2,27)$ \\
\hline DRFe & $2(11,11)$ & $1(7,69)$ & $18(37,50)$ & $20(35,09)$ & $19(55,88)$ & $26(59,10)$ \\
\hline
\end{tabular}

$\mathrm{Hb}=$ Hemoglobina, RFeN= Reservas de hierro normales, RIFe= Riesgo de depleción de las reservas de hierro,

$\mathrm{DRFe}=$ Depleción de las reservas corporales de hierro, Los valores en paréntesis representan el porcentaje. 
ferritina sérica que tiene correspondencia con el almacenamiento de hierro en los tejidos; posteriormente disminuye el hierro sérico a medida que se agotan las reservas y esto sucede, por lo general, antes de que se afecte la producción de hemoglobina $(33,38)$. En nuestra investigación, tanto la disminución de ferritina como hierro sérico denotan un inadecuado estado corporal de este nutriente en gestantes que habitan en las zonas rurales del estado Zulia, Venezuela. En consecuencia, la baja ingesta de hierro es consistente con la alta prevalencia de anemia y afectación de las reservas de hierro detectadas en esta investigación, en la cual las adolescentes gestantes presentan anemia con afectación de las reservas corporales de hierro en 45,79\%; igualmente, afectación de las reservas corporales de hierro sin anemia en 30,37\%, apreciándose anemia con reservas corporales de hierro normal en sólo 9,34\% de las gestantes.

Es pertinente señalar, que la prevalencia de anemia con afectación de las reservas corporales de hierro reportada en nuestra investigación fue superior a la reportada por Méndez y col. (2009) en adolescentes gestantes mexicanas (7,1\%) (39). No obstante, la prevalencia de afectación de las reservas corporales de hierro sin anemia resultaron más baja $(55,5 \%)$ que la reportada por los mismos autores. Estudios en siete países latinoamericanos señalan que 39\% de las embarazadas padece anemia y $48 \%$ de éstas anemias presentan afectación de las reservas corporales de hierro (40).

En el presente estudio, la vulnerabilidad orgánica para presentar anemia con afectación de las reservas corporales de hierro es manifiesta por el hecho de que este grupo de adolescentes en su mayoría enfrenta por vez primera un embarazo; sin embargo, esta prevalencia debe considerarse en relación con otros factores, como hemodilución asociada con el embarazo, aumento en las necesidades de hierro para reponer las pérdidas basales e incremento de la masa eritrocitaria para satisfacer el crecimiento fetal y placentario $(37,40)$. Otro aspecto a considerar representa el hábito de alimentación; es decir, el consumo de frituras procesadas con alto contenido de hidratos de carbono, grasas saturadas, saborizantes y gaseosas (consumo de productos "chatarra"), una característica común en una de cada dos adolescentes independientemente de que ellas mismas los consideren nocivos (41). Este argumento, resulta de gran importancia si consideramos que las adolescentes embarazadas representan un grupo vulnerable para la malnutrición tanto por su estado puberal como por la presencia del producto en gestación, ambos en riesgo de déficit de macro y micronutrientes.

Al respecto, el consumo de hierro comprobado por nuestra investigación en adolescentes embarazadas de zonas rurales fue significativamente más bajo, considerando el hábito de alimentación como un factor que aumenta la probabilidad de modificación de las reservas corporales de hierro y predisposición conjunta para la ocurrencia de anemia. En este contexto, la prevalencia de anemia con afectación de las reservas de hierro observada fue mayor en las adolescentes gestantes urbanas al compararlas con las rurales durante el III trimestre, seguido por el II y I trimestre. Si bien, el bajo consumo de hierro es uno de los factores asociados con anemia y afectación de las reservas de hierro en la muestra analizada, un estudio previo en Estados Unidos demostró que aún cuando la ingesta de hierro fue similar entre grupos de mujeres México-Americanas y mujeres blancas no Hispánicas (económicamente más favorecidas en teoría), la prevalencia de anemia por deficiencia de hierro fue más alta en el primer grupo $(6,2 \%$ vs $2,3 \%)$, debido a la presencia de factores relacionados con el ingreso familiar y la elevada ingesta de alimentos que pueden alterar la absorción del hierro (42).

Contrario a lo observado en la prevalencia de anemia con afectación de las reservas de hierro en gestantes analizadas en este estudio, la prevalencia de afectación de las reservas de hierro sin anemia, fue superior en las adolescentes gestantes procedentes de la zona rural en el I y II trimestre comparadas con gestantes de la zona urbana; sin embargo, durante el III trimestre la prevalencia fue mayor en adolescentes gestantes urbanas $(29,42 \%)$, que en las procedentes de la zona rural $(22,7 \%)$.

Evidentemente la mayor parte de los estudios asocian la anemia con efectos desfavorables tanto para la madre como para el hijo durante y después del embarazo, no menos importantes son las reservas corporales de hierro, dado que es precisamente este mineral circulante en la madre, unido a la transferrina, el que capta los receptores de esta proteína en la placenta, para transferirlo al feto (43). Al respecto, existe evidencia de la relación entre el estado del hierro materno y el fetal, aún cuando una regulación positiva en la expresión de proteínas de transporte de hierro puede proteger al feto de la deficiencia materna, (33).

Durante el embarazo, la exigencia corporal de hierro materno incrementa en promedio aproximadamente a 1.000 $\mathrm{mg}$. Esta cantidad cubre $350 \mathrm{mg}$ asociado con el crecimiento del feto y la placenta, $500 \mathrm{mg}$ que corresponden con la expansión de la masa de glóbulos rojos, y $250 \mathrm{mg}$ relacionados con la pérdida de sangre durante el parto. En consecuencia, el aumento de las necesidades debe ser apoyado por el aumento de la ingesta de hierro materna, incrementado $6 \mathrm{mg} /$ día en el I trimestre, $19 \mathrm{mg} /$ día en el II trimestre, a $22 \mathrm{mg} /$ día en el III trimestre del embarazo. Con el fin de cumplir con estos requisitos cada vez mayores, la embarazada debe recurrir a las reservas corporales de hierro, aumentando el riesgo de deficiencia de hierro (44). En la gestación, las mayores necesidades de hierro se sitúan principalmente en el III trimestre. No obstante, el metabolismo del hierro está regulado por múltiples mecanismos que intentan compensar el aumento de las necesidades de hierro o situaciones de deficiencia con incrementos en la absorción intestinal de este mineral (45). Por ende, dadas las mayores necesidades de hierro en el III trimestre de la gestación y la respuesta del metabolismo del hierro para compensar el riesgo de deficiencia (36), es probable que mujeres con bajo consumo de hierro en la dieta antes o durante el embarazo presenten depleción de las reservas corporales de hierro en cualquier trimestre del embarazo sin presencia de anemia y no asociada con la procedencia urbana o rural, propuesta en esta investigación.

En este contexto, los enfoques basados en la alimentación para aumentar el aporte de hierro mediante la fortificación de alimentos y la diversificación alimentaria son estrategias importantes para mantener las reservas adecuadas de hierro, reduciendo el riesgo que representa la deficiencia de hierro y anemia para las adolescentes embarazadas y sus productos. Un aspecto trascendental a considerar junto con la suplementación de macro y micronutriente, son los programas nutricionales desde edades tempranas de la vida, en especial entre las niñas que postergan su niñez para convertirse en madres prematuras, con el objeto de promover una nutrición adecuada que permita un mejor desarrollo para que inicien su vida reproductiva en las mejores condiciones de salud.

En conclusión, aunque se considere la suma de estrategias que favorezcan la nutrición adecuada durante la gestación prematura, la prevención del embarazo en la población ado- 
lescente en países desarrollados o en vías de desarrollo, debe centrarse en programas educativos que alienten a nuestros niños a seguir un patrón cultural que facilite la vivencia de sus diferentes etapas de vida, contribuyendo con un verdadero desarrollo social, cultural y por resultado de prosperidad económica, incrementando la probabilidad de una mayor concentración de núcleos familiares consolidados en un país indudablemente progresista.

\section{RESUMEN}

Objetivo: Analizar la prevalencia de anemia y depleción de las reservas corporales de hierro (DRFe) en adolescentes gestantes urbanas (U) y rurales (R). Metodología: Fueron estudiadas 214 adolescentes gestantes $(U=100$ y $R=114)$, de baja condición socio-económica. Se realizó una evaluación clínica-obstétrica, nutricional y bioquímica. Según recomendaciones de la OMS y el Grupo Consultivo Internacional de Anemia se consideró anemia en gestantes, I y III trimestre $\mathrm{Hb} \leq 110 \mathrm{~g} / \mathrm{L}$, y II trimestre $\mathrm{Hb} \leq 105 \mathrm{~g} / \mathrm{L}$. DRFe= ferritina $<15 \mathrm{ug} / \mathrm{L}$; reservas insuficientes de hierro(RIFe)=ferritina 15-20ug/L y reservas de hierro normal (RFe normal)=ferritina $>20 u g / L$. Se consideró significancia estadística $p<0,05$. Resultados: En las adolescentes gestantes la prevalencia de anemia con afectación de las reservas corporales de hierro (RIFe+DRFe) fue 45,79\% ( rurales $=22,90 \%$ vs urbanas $=22,89 \%$ ), afectación de las reservas corporales de hierro (RIFe + DRFe) sin anemia 30,37\% ( $R=17,29 \%$ vs. $U=13,08 \%)$ y anemia con reservas corporales de hierro normal $9,34 \%$ ( $R=6,07 \%$ vs. $U=3,27 \%$ ); mostrando asociación estadísticamente significativa para la zona urbana $(x 2=8,1282 p=0,0172)$ y la zona rural $(x 2=11,6270$ $p=0,0030)$. Conclusiones: La anemia ferropénica es una complicación frecuente en adolescentes gestantes rurales y urbanas, asociada a malos hábitos alimentarios propios de la edad. Es necesario, incorporar estrategias de educación y recuperación nutricional en la atención primaria de salud y programas de salud maternoinfantil.

Palabras clave: Anemia, deficiencia de hierro, adolescente, gestación.

\section{BIBLIOGRAFÍA}

1. World Health Organization Department of Nutrition for Health and Development/United Nations University/UNICEF. Iron deficiency anemia, assessment, prevention and control: a guide for programme managers. Geneva: WHO, 2001. Disponible en: http://www.who.int/nutrition/ publications/micronutrients/anaemia_iron_deficiency WHO_NHD_01.3/en/index.html), Revisado 07-12-2011

2. World Health Organization, Centers for Disease Control and Prevention. Assessing the iron status of populations. Geneva: WHO, 2007. (pp108). Disponible en: http:// whalibdoc.who.int/publications/2004/9241593156_eng. pdf. Revisado 07-12-2011

3. Montoya Sáez P. ALIMENTACIÓN, NUTRICIÓN Y SALUD. Prosalud. Salud y desarrollo.(pp31). Disponible en: htp:// www.fao.org/righttofood/KC/downloads/vl/docs/AH420. pdf Revisado 07-12-2011. Revisado 07-12-2011

4. López-Blanco M. Carmona A. La transición alimentaria y nutricional: un reto en el siglo XXI, An Venez Nutr 2005; 18:90-104.

5. Laurentin A. Schnell M. Tovar J. Domínguez Z. Pérez B. López de Blanco M. Transición alimentaria y nutricional: Entre la desnutrición y la obesidad, An Venez Nutr 2007; 20 (1): 47-52.

6. Solano L. Barón M. del Real S. Situación nutricional de preescolares, escolares, y adolescentes de Valencia, Carabobo, Venezuela, An Venez Nutr 2005; 18(1):72-6.

7. García-Casal M. La deficiencia de hierro como problema de Salud Pública, An Venez Nutr 2005; 18: 45-8.

8. World Health Organization. The prevalence of anaemia in women: a tabulation of available information. 2nd ed. Geneva: World Health Organización, 1992.

9. Novoa E. Anemia en la adolescencia, un desafío diagnóstico y terapéutico, Revista del Anemia Working Group Latin America (AWGLA) 2006; 2: 3-10. http://www.awgla.com publicaciones/descargas/CompendioGuias.pdf. Revisado 07-12-2011.

10. Osório M. Determinant factors of anemia in children, J Pediatr (Rio J) 2002; 78: 269-78.

11. Monteagudo E. Cabo T. Dalmau J. Anemias nutricionales en el adolescente, Acta Pediatr Esp 2000; 58: 594-600. Disponible en: http://WWw.gastroinf.com/SecciNutri] ANEMIAS.pdf. Revisado 07-12-2011

12. World Health Organization. Adolescent pregnancy-Unmet needs and undone deeds. A review of the literature and programmes Issues in Adolescent Health and Development. 2007. ISBN 978924159565 0. Disponible en: http:// whqlibdoc.who.int/publications/2007/9789241595650 eng.pdf. Revisado 07-12-2011.

13. Milman N. Iron in pregnancy - how do we secure an appropriate iron status in the mother and child?, Ann Nutr Metab 2011;59(1):50-4.

14. Rodríguez Ganen O. Fernández Monagás S. Gazapo Pernas R. Fernández Manzano E. Rodríguez Acosta T. Sánchez Salazar R. Castanedo Valdés R. Cutié León E. Factores que inciden en la anemia ferropénica de la embarazada, Rev Cubana Farm 2002;36(3):176-81.

15. Olausson PO. Cnattingius S. Haglund B. Teenage pregnancies and risk of late fetal death and infant mortality, Br J Obstet Gynaecol. 1999; 106(2):116-21.

16. Allen LH. Anemia and iron deficiency: effects on pregnancy outcome, Am J Clin Nutr 2000; 71:1280S-4S.

17. León P. Minassian M. Borgoño R. Bustamante F. Embarazo Adolescente, Rev. Ped. Elec. [en línea] 2008; 5(1): 42-51.

18. Forrellat M. Gautier du Défaix H. Fernández N. Metabolismo del hierro, Rev Cubana Hematol Inmunol Hemoter 2000; 16(3): 149-60.

19. Distribución Espacial y Urbanización de la Población en América Latina y el Caribe (DEPUALC). Definición de población rural y urbana. Criterios empleados por diversos países de América Latina y el Caribe. Criterios empleados en Venezuela en los Censos de 1961, 1971, 1981 y 1990. En CEPAL, Boletín Demográfico N63, de enero de 1999. p 1-4 Disponible en: (http://www.eclac.cl/celade/publica/ LCR1999/LCR1999def00e.htm). (Revisado 07-12-2011).

20. Méndez-Castellano H. Méndez MC. Estratificación social y biología humana, Arch Venez Puer Ped 1986; 49:93-104.

21. Landaeta Jiménez M., López Blanco M., Méndez Castellano H. (1989). Area Muscular y área grasa. Estudio transversal de Caracas. Arch Venez Puer Pediatr 52: 97-106.

22. Organización Panamericana de la Salud (OPS). Organización Mundial de la Salud (OMS). Promoción del crecimiento y desarrollo integral de niños y adolescentes. Módulo de aprendizaje aiepi. Serie HCT/AIEPI - 25.E.1. 1999; 142.

23. Ministerio de Sanidad y Asistencia Social. Instituto Nacional de Nutrición. Dirección Técnica: División de Investigación en Alimentos. Tabla de composición de alimentos para uso práctico. Revisión 1999. Publicación $N^{\circ}$ 52. Serie de 
Cuadernos Azules. Caracas-Venezuela. 1999.

24. Landaeta-Jiménez M. Pérez BM. Escalante Y. Adiposidady patrón de grasa en jóvenes venezolanos por estrato social, Arch Latinoam Nutr 2002; 52:128-36.

25. Instituto Nacional de Nutrición. Tabla de composición de alimentos para uso práctico. Revisión 1999. Publicación $N^{\circ}$ 52. Serie Cuadernos Azules. Caracas - Venezuela. p. 97, 1999.

26. Nestel P. Davidsson L. Anemia, Deficiencia de Hierro y Anemia Ferropriva. Grupo Consultor Internacional de Anemia Nutricional (INACG). Oficina de Salud, Enfermedades Infecciosas y Nutrición, Oficina de Salud Global, Agencia para el Desarrollo Internacional de los Estados Unidos (USAID), Junio 2004.

27. Van den Broek NR. Letsky EA. Etiology of anemia in pregnancy in south Malawi, Am J Clin Nutr. 2000; 72 (Suppl): 247S-56S.

28. Beard J. Piñero D. Metabolismo del hierro. En: O'Donnell A, Viteri F, Carmuega E. Deficiencia de Hierro. Desnutrición Oculta en América Latina. Centro Asociado de La Facultad de Medicina de la Universidad del Salvador. Centro de Estudios Sobre Nutrición Infantil. CESNI. 2003; 13-47.

29. Villarroel Rosende G. Embarazo adolescente y pobreza rural. Revista Digital e Rural, Educación, cultura y desarrollo rural. Año $4 N^{\circ} 7$ junio 2006, ISSN 0717-9898 http:// WWw.revistaerural.cl/EmbarazoGVillarroel.pdf. Revisado 05-01-2012.

30. Murillo S. Ulate E. Mata L. Nutrición materna durante el embarazo: estudio de mujeres de una zona rural de Costa Rica. Bol Oficina Sanit Panam 1988; 104 (4)345-54.

31. Coll A. Reproducción en la adolescencia. En: Atención Integral de Adolescentes y Jóvenes. Volumen I: Adolescencia Normal. Buenos Aires: Sociedad Argentina de Pediatría.1989. p 203-18.

32. Rodríguez J. Reproducción en la adolescencia: el caso de Chile y sus implicaciones de política, Rev CEPAL 2005; 86: 123-46.

33. Parra Sosa B. Restrepo M S. Manjarrés Correa L. Mancilla López L. Indicadores bioquímicos del hierro materno en el tercer trimestre de la gestación y su relación con la antropometría materna y el peso al nacer, IATREIA 2009; 22(1): 16-26.
34. Restrepo M S. Mancilla L. Parra B. Manjarrés L. Zapata N. Restrepo Ochoa P. Martínez M. Evaluación del estado nutricional de mujeres gestantes que participaron de un programa de alimentación y nutrición. Rev Chil Nutr 2010; 37(1): 18-30.

35. Vila M. Quintana M. Ingesta de hierro dietario en mujeres adolescentes de instituciones educativas. An Fac Med 2008; 69(3):172-5.

36. Ribot B. Aranda N. Arija V. Suplementación temprana o tardía: similar evolución del estado de hierro durante el embarazo, Nutr Hosp 2012; 27(1):219-26.

37. Iglesias J. Tamez L. Reyes I. Anemia y embarazo, su relación con complicaciones maternas y perinatales, Medicina Universitaria 2009; 11 (43): 95-8.

38. Gambling L. Kennedy C. McArdle HJ. Iron and copper in fetal development, Semin Cell Dev Biol 2011; 22(6):637-44.

39. Méndez Estrada R. Pacheco B. Noriega Verdugo H. Quihui L. Morales G. Valencia M. Prevalencia de deficiencia de hierro y de anemia por deficiencia de hierro en adolescentes embarazadas del noroeste de México 2007-2008, Arch Latinoam Nutr 2009; 59(2):147-51.

40. Vera Gamboa L. Quintal Duarte R. González Martínez P. Vázquez Castillo G. Prevalencia de anemia ferropénica en mujeres embarazadas rurales en Valladolid, Yucatán, México, Ginecol Obstet Mex 2009; 77(12):544-49.

41. Herrera-Suarez C. Vásquez-Garibay E. Romero-Velarde E. Romo-Huerta H.P., Garcia D., Troyo-Sanroman R. Hábitos de alimentación y factores culturales en adolescentes embarazadas, Arch Latinoam Nutr 2008; 58 (1):19-26.

42. Frith-Terhune A. Cogswell M. Kettel Khan L. Will J. Ramakrishnan U. Iron deficiency anemia: higher prevalence in Mexican American than in non-Hispanic white females in the third National Health and Nutrition Examination Survey, 1988-1994, Am J Clin Nutr 2000;72(4):963- 8.

43. McArdle HJ. Lang C. Hayes H. Gambling L. Role of the placenta in regulation of fetal iron status, Nutr Rev 2011; 69 (Suppl 1):S17-22.

44. Scholl TO. Maternal iron status: relation to fetal growth, length of gestation, and iron endowment of the neonate, Nutr Rev 2011; 69 (Supp/ 1):S23-9.

45. Beard J. Han O. Systemic iron status, Biochim Biophys Acta 2009; 1790 (7):584-8. 